\title{
Design, Synthesis, and Antimicrobial Activity, of New 1,4-disubstituted Octahydroquinoxaline-2,3-dione Derivatives
}

\author{
Mostafa A. Hussein ${ }^{*}$ \\ Department of Pharmaceutical Organic Chemistry, Faculty of Pharmacy, Assiut University, Assiut-71526, Egypt. \\ "E-mail:mostafa1705@yahoo.com \\ Received November 15, 2010, Accepted March 7, 2011
}

\begin{abstract}
A series of 1,4-disubstituted octahydroquinoxaline-2,3-dione derivatives was prepared through two steps reaction. The latter involves the formation of $N, N$-disubstituted cyclohexane-1,2-diamine derivatives (la-j) through reductive alkylation of 1,2-cyclohexanediamine with different aldehydes in presence of sodium cyanoborohydride. Fusion of compounds (1a-j) with diethyl oxalate affording the target compounds (2a-j). Elucidation of structures of compounds (2a-j) was based upon different spectral data as well as the elemental methods of analyses. In addition, mass spectrometry and X-ray diffraction analyses were carried out. Moreover, the lipophilicity of the target compounds as expressed from the Clog P. Most of the test compounds (2a-j) showed weak to moderate antibacterial and antifungal activities against most of the used bacterial and fungal strains in comparison to chloramphenicol and clotrimazole as reference drugs respectively.
\end{abstract}

Key Words : Antimicrobial, Activity, Octahydroquinoxaline-2,3-dione, Diffraction, Synthesis

\section{Introduction}

The emergence of microbial resistance is an evolutionary process based on selection for organisms that have enhanced ability to survive doses of antibiotics that would have previously been lethal. Survival of bacteria often results from an inheritable resistance. ${ }^{1,2}$ Moreover, antibiotic resistance may impose a biological cost and consequently, spread of antibiotic resistant bacteria may be hampered by reduced fitness associated with the resistance. However, additional mutations may compensate for this fitness cost and aids the survival of this bacteria. ${ }^{3-6}$ Hence, the search for new and potent antimicrobial agents is gaining interest. On the other hand, nitrogen containing heterocycles are indispensable structural units for medicinal chemists. Among the various heterocyclic compounds, quinoxalines form an attractive biologically active molecules as those form part of various antibiotics such as echinomycin, levomycin and actinoleutin ${ }^{7}$ that are known to possess other biological potentials such as adenosine receptor antagonist, anticancer, anthelimintic, antidepressant, anti-inflammatory, and antituberular activities. ${ }^{8-13}$ Moreover, quinoxaline and its analogues have been investigated as the catalyst's ligands. ${ }^{14}$ In view of the literature regarding antimicrobial potency of quinoxalines and their mode of action that prevent DNA-directed RNA synthesis by virtue of binding to $\mathrm{CpG}$ site on DNA, the quinoxaline nucleus is focused on synthesizing newer derivatives to explore potent antimicrobial activities. ${ }^{4,8}$

The continued interest in designing new flexible quinoxaline and quinoxalinedione molecules stems mainly because of the outstanding biological activities exhibited by several derivatives incorporating such heterocyclic moieties. ${ }^{15-17}$ Accordingly, the present work aims to the design and synthesis of new octahydroquinoxalinedione derivatives and elucidation of their structures, in addition to, testing the target compounds for their expected antimicrobial effects, if any.

\section{Experimental}

Chemistry. Melting points were determined in an electrothermal melting point apparatus (Stuart Scientific, Staffordshine, STIS, SMP3, UK) and were uncorrected. Monitoring of the chemical reactions was carried out by TLC using precoated silica gel plates (kieselgel $0.25 \mathrm{~mm}, 60 \mathrm{G} \mathrm{F} 254$, Merck, Germany) and $\mathrm{CHCl}_{3} / \mathrm{CH}_{3} \mathrm{OH}$ as the mobile phase. Visualization of the spots was effected by ultraviolet lamp (model CM-10, USA) at wavelengths 254 and $365 \mathrm{~nm}$ and/ or iodine stain.

IR spectra were carried out as $\mathrm{KBr}$ discs on a Shimadzu IR-470 Spectrometer (Shimadzu, Japan). ${ }^{1} \mathrm{H}-\mathrm{NMR}$ spectra were scanned on a Varian EM-360L NMR spectrometer (60 $\mathrm{MHz}$, Varian, USA). Chemical shifts are expressed in $\delta$ values (ppm) relative to TMS as an internal standard; using $\mathrm{CDCl}_{3}$ as a solvent and $\mathrm{D}_{2} \mathrm{O}$ was used for the detection of the exchangeable protons.

Electron Impact mass spectra (EI-MS) were run with JEOL JMS600 mass spectrometer at $70 \mathrm{eV}$ (Thermo Electron Corporation, Japan) at the Micro Analytical Central Lab, Assiut University. Elemental microanalyses were performed on a Perkin-Elmer, 240 Elemental Analyzer, at the Unit of Microanalysis, Assiut University and the Micro Analytical Center, Faculty of Science, Cairo University. X-ray diffraction (XRD) was carried out using XRD unit, at Dept., of physics, Faculty of Science, Assiut University.

$N, N$-Disubstituted Cyclohexane-1,2-diamine Derivatives (1a-j). Sodium cyanoborohydride $(48.0 \mathrm{mmol})$ was added portion wise to a solution of the appropriate aldehyde 


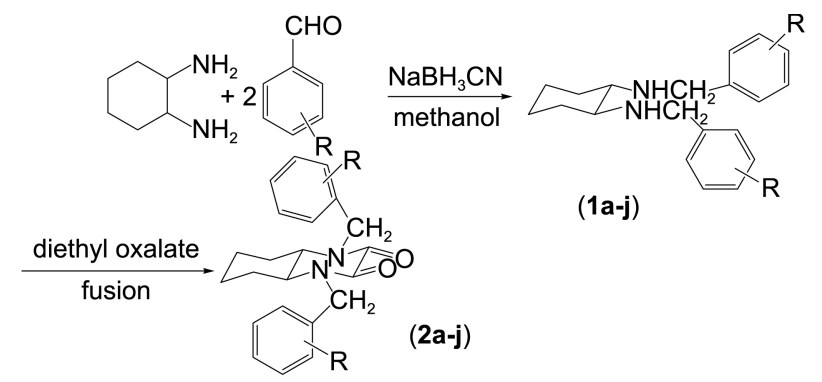

$\mathrm{R}=(\mathbf{a}) \mathrm{H}$, (b) $p-\mathrm{Br}$, (c) $p-\mathrm{Cl}$, (d) $o-\mathrm{Cl},(\mathbf{e}) p-\mathrm{CH}_{3}$, (f) $p-\mathrm{C}_{3} \mathrm{H}_{7}$, (g) $p-\mathrm{OCH}_{3}$, (h) $\mathrm{NMe}_{2}$, (i) $p-\mathrm{F}$, and (j) $o, m-\mathrm{OCH}_{2} \mathrm{O}$

Scheme 1. Synthesis of compounds $\mathbf{1 a}-\mathbf{j}$ and $\mathbf{2 a - j}$.

derivative $(60 \mathrm{mmol})$ and 1,2-cyclohexylamine $(30 \mathrm{mmol})$ in methanol $(70 \mathrm{~mL})$ at $0{ }^{\circ} \mathrm{C}$. The reaction mixture was stirred for $3 \mathrm{~h}$ at ambient temperature, an additional amount of sodium cyanoborohydride $(8.1 \mathrm{mmol})$ was added portion wise and stirring was continued for further $2 \mathrm{~h}$. The reaction mixture was quenched with distilled water, concentrated and extracted with chloroform; the combined organic extract was washed with distilled water, and dried. Concentration and column chromatography $\left(\mathrm{CHCl}_{3} / \mathrm{CH}_{3} \mathrm{OH}\right)$ afforded compounds (1a-j) as pale yellow oils, Scheme 1. Yields and ${ }^{1} \mathrm{HNMR}$ data are given in Table 1.

1,4-Disubstituted Octahydroquinoxaline-2,3-dione (2a-j). A mixture of the $N, N$-disubstituted cyclohexane-1,2-diamine derivatives (1a-j) $(7.5 \mathrm{mmol})$, and freshly distilled diethyl oxalate $(7.5 \mathrm{mmol})$ was fussed for $2 \mathrm{~h}$, treated with ether $(30 \mathrm{~mL})$ and left overnight. The product was filtered, dried and crystallized from absolute ethanol, Scheme 1, Tables 2, and 3.

Calculation of the $\log \mathbf{P}$ Values. The $\log P$ values of the target compounds $(\mathbf{2} \mathbf{a}-\mathbf{j})$, were computed with a routine method called calculated $\log \mathrm{P}(\mathrm{Clog} \mathrm{P})$ contained in a PCsoftware package (McLogP 2.0, BioByte Corp., CA, USA).
A representation of the molecular structure where hydrogens were omitted or 'suppressed' (SMILES notation) was entered into the program, which computes the $\log \mathrm{P}$ based on Crippen's fragmentation ${ }^{18}$, and the results are given in Table 2.

X-ray Diffraction. X-ray diffraction (XRD) was carried out using XRD unit, model PW1710 control unit, PW1710 generator and PW1050 Goniometer, Anode material $\mathrm{Cu}$, Optics automatic divergence slit 0.1 and beta filtering graphite monochromator and software visualx, traces and PDF2 $1999^{19}$ at Dept., of physics, Faculty of Science, Assiut University.

\section{Antimicrobial Activity.}

Antibacterial Activity: The synthesized compounds (2a-j) were tested for their antibacterial activity in vitro, in comparison with chloramphenicol as a reference drug using the standard agar cup diffusion method ${ }^{20}$ using six bacterial species representing both Gram-positive and Gram-negative strains at the Assiut University Mycological Center (AUMC). The strains are common contaminants of the environment in Egypt and some of which are involved in human and animal diseases. The used bacterial strains are Serratia marscens (AUMC B55), Pseudomonas aeruginosa (AUMC B73), and Escherichia coli (AUMC B53) as representatives for the Gram negative strains, while the Gram positive strains were represented by Staphylococcus aureus (AUMC B54), Bacillus cereus (AUMC B52), and Micrococcus luteus (AUMC $\mathrm{B} 112)^{21,22}$

Bacterial strains were individually cultured for $48 \mathrm{~h}$ in $100 \mathrm{~mL}$ conical flasks containing $30 \mathrm{~mL}$ Nutrient Agar (NA) medium. Bioassay was done in $10 \mathrm{~cm}$ sterile plastic Petri dishes in which One $\mathrm{mL}$ suspension and $15 \mathrm{~mL}$ of NA were poured. Plates were shaken gently to homogenize the inocula.

After solidification of the media, $5 \mathrm{~mm}$ cavities were cut in the solidified agar ( 4 cavities/plate) using sterile cork

Table 1. Yields and ${ }^{1} \mathrm{HNMR}$ data of compounds (1a-j)

\begin{tabular}{|c|c|c|c|}
\hline $\begin{array}{l}\text { Compd. } \\
\text { No. }\end{array}$ & $\mathrm{R}$ & $\begin{array}{c}\text { Yield } \\
\%\end{array}$ & ${ }^{1} \mathrm{H} \mathrm{NMR}$ \\
\hline $1 \mathbf{a}$ & $\mathrm{H}$ & 85 & 1.00-2.40 (m, 12H, c-hexyl, and 2NH), 3.30-3.90 (dd, 4H, 2CH $)$, and $7.20(\mathrm{~s}, 10 \mathrm{H}, 2 \mathrm{Ph})$. \\
\hline $1 \mathbf{b}$ & $p-\mathrm{Br}$ & 95 & 0.80-2.20 (m, 12H, c-hexyl, and 2NH), 3.30-4.00 (dd, 4H, 2CH $)$, and 6.90-7.5 (dd, 8H, $2 \mathrm{Ph})$ \\
\hline $1 \mathrm{c}$ & $p-\mathrm{Cl}$ & 93 & 0.85-2.25 (m, 12H, c-hexyl, and 2NH), 3.33-3.90 (m, 4H, 2CH $)$, and $7.15(\mathrm{~s}, 8 \mathrm{H}, 2 \mathrm{Ph})$. \\
\hline $1 d$ & $o-\mathrm{Cl}$ & 67 & 0.85-2.40 (m, $12 \mathrm{H}, c$-hexyl, and 2NH), 3.15-4.10 (m, 4H, 2CH $)$, and 6.70-7.40 (m, $8 \mathrm{H}, 2 \mathrm{Ph})$. \\
\hline 1e & $p-\mathrm{CH}_{3}$ & 85 & $0.85-2.20(\mathrm{~m}, 12 \mathrm{H}, c$-hexyl, and $2 \mathrm{NH}), 2.33\left(\mathrm{~s}, 6 \mathrm{H}, 2 \mathrm{CH}_{3}\right), 3.30-4.00\left(\mathrm{~m}, 4 \mathrm{H}, 2 \mathrm{CH}_{2}\right)$, and $7.15(\mathrm{~s}, 8 \mathrm{H}, 2 \mathrm{Ph})$ \\
\hline 1f & $p-i-\mathrm{C}_{3} \mathrm{H}_{7}$ & 86 & $\begin{array}{l}0.85-2.10\left(\mathrm{~m}, 24 \mathrm{H}, c \text {-hexyl, } 4 \mathrm{CH}_{3} \text {, and } 2 \mathrm{NH}\right), 2.50-3.00(\mathrm{~m}, 2 \mathrm{H}, 2 \mathrm{CH}), 3.35-400\left(\mathrm{~m}, 4 \mathrm{H}, 2 \mathrm{CH}_{2}\right) \text {, and } 7.10(\mathrm{~s} \text {, } \\
8 \mathrm{H}, 2 \mathrm{Ph}) .\end{array}$ \\
\hline $1 \mathrm{~g}$ & $p-\mathrm{OCH}_{3}$ & 90 & 0.70-2.30 (m, 12H, c-hexyl, and 2NH), 3.30-3.90 (m, 10H, $2 \mathrm{CH}_{2}$, and $\left.2 \mathrm{CH}_{3}\right)$, and 6.80-7.30 (m, $\left.8 \mathrm{H}, 2 \mathrm{Ph}\right)$. \\
\hline $1 \mathrm{~h}$ & $p-\mathrm{N}\left(\mathrm{CH}_{3}\right)_{2}$ & 92 & 0.85-2.35 (m, 12H, c-hexyl, and 2NH), $2.85\left(\mathrm{~s}, 12,4 \mathrm{CH}_{3}\right), 3.25-3.85\left(\mathrm{~m}, 4 \mathrm{H}, 2 \mathrm{CH}_{2}\right)$, and 6.40-7.20 (m, $\left.8 \mathrm{H}, 2 \mathrm{Ph}\right)$. \\
\hline $1 \mathbf{i}$ & $p-\mathrm{F}$ & 90 & $0.85-2.30(\mathrm{~m}, 12 \mathrm{H}, c$-hexyl, and 2NH), 3.30-4.00 (m, 4H, 2CH $)$, and 6.60-7.33 (m, 8H, 2Ph) \\
\hline $1 \mathbf{j}$ & $o, m-\mathrm{OCH}_{2} \mathrm{O}$ & 90 & $\begin{array}{l}0.85-2.33(\mathrm{~m}, 12 \mathrm{H}, c \text {-hexyl, and } 2 \mathrm{NH}), 3.20-3.85\left(\mathrm{~m}, 4 \mathrm{H}, 2 \mathrm{CH}_{2}\right), 5.85\left(\mathrm{~s}, 4 \mathrm{H}, 2 \mathrm{CH}_{2} \text { of pripeonyl), and } 6.50-\right. \\
6.85(\mathrm{~m}, 6 \mathrm{H}, 2 \mathrm{Ph}) .\end{array}$ \\
\hline
\end{tabular}


Table 2. The physicochemical data and elemental microanalyses of compounds $\mathbf{2 a - j}$

\begin{tabular}{|c|c|c|c|c|c|c|c|c|c|}
\hline $\begin{array}{l}\text { Compd } \\
\text { No. }\end{array}$ & $\mathrm{R}_{1}$ & $\begin{array}{l}\text { M. formula } \\
\text { (M. Wt) }\end{array}$ & $\begin{array}{c}\text { Yield }^{a} \\
\%\end{array}$ & $\begin{array}{l}\mathrm{mp} \\
{ }^{\circ} \mathrm{C}\end{array}$ & $\mathrm{R}_{\mathrm{f}}^{b}$ & $\mathrm{C} \log \mathrm{P}$ & \multicolumn{3}{|c|}{$\begin{array}{c}\text { CHN } \\
\text { Found/Cacd }\end{array}$} \\
\hline $2 a$ & $\mathrm{H}$ & $\mathrm{C}_{22} \mathrm{H}_{24} \mathrm{~N}_{2} \mathrm{O}_{2}(348.44)$ & 82 & $100-2$ & 0.42 & 5.57 & $\begin{array}{l}75.57 \\
75.83\end{array}$ & $\begin{array}{l}7.26 \\
6.94\end{array}$ & $\begin{array}{l}7.86 \\
8.04\end{array}$ \\
\hline $2 b$ & $p-\mathrm{Br}$ & $\mathrm{C}_{22} \mathrm{H}_{22} \mathrm{Br}_{2} \mathrm{~N}_{2} \mathrm{O}_{2}(506.23)$ & 90 & 170 & 0.41 & 7.3 & $\begin{array}{l}52.09 \\
52.20\end{array}$ & $\begin{array}{l}4.02 \\
4.38\end{array}$ & $\begin{array}{l}5.86 \\
5.53\end{array}$ \\
\hline $2 c$ & $p-\mathrm{Cl}$ & $\mathrm{C}_{22} \mathrm{H}_{22} \mathrm{Cl}_{2} \mathrm{~N}_{2} \mathrm{O}_{2}$ (417.33) & 90 & 173 & 0.52 & 7.0 & $\begin{array}{l}63.77 \\
63.32\end{array}$ & $\begin{array}{l}5.36 \\
5.31\end{array}$ & $\begin{array}{l}6.61 \\
6.71\end{array}$ \\
\hline 2d & $o-\mathrm{Cl}$ & $\mathrm{C}_{22} \mathrm{H}_{22} \mathrm{Cl}_{2} \mathrm{~N}_{2} \mathrm{O}_{2}$ (417.33) & 87 & 170 & 0.58 & 7.0 & $\begin{array}{l}63.00 \\
63.32\end{array}$ & $\begin{array}{l}5.54 \\
5.31\end{array}$ & $\begin{array}{l}6.51 \\
6.71\end{array}$ \\
\hline $2 e$ & $p-\mathrm{CH}_{3}$ & $\mathrm{C}_{24} \mathrm{H}_{28} \mathrm{~N}_{2} \mathrm{O}_{2}$ (376.49) & 75 & 152 & 0.32 & 6.57 & $\begin{array}{l}76.03 \\
76.56\end{array}$ & $\begin{array}{l}7.77 \\
7.50\end{array}$ & $\begin{array}{l}7.19 \\
7.44\end{array}$ \\
\hline $2 f$ & $p-i-\mathrm{C}_{3} \mathrm{H}_{7}$ & $\mathrm{C}_{28} \mathrm{H}_{36} \mathrm{~N}_{2} \mathrm{O}_{2}(432.60)$ & 80 & 150 & 0.52 & 8.42 & $\begin{array}{l}77.35 \\
77.74\end{array}$ & $\begin{array}{l}8.74 \\
8.39\end{array}$ & $\begin{array}{l}6.91 \\
6.48\end{array}$ \\
\hline $2 \mathrm{~g}$ & $p-\mathrm{OCH}_{3}$ & $\mathrm{C}_{24} \mathrm{H}_{28} \mathrm{~N}_{2} \mathrm{O}_{4}(408.49)$ & 85 & 158 & 0.35 & 5.41 & $\begin{array}{l}70.36 \\
70.57\end{array}$ & $\begin{array}{l}7.19 \\
6.91\end{array}$ & $\begin{array}{l}6.63 \\
6.86\end{array}$ \\
\hline $2 \mathrm{~g}$ & $p-\mathrm{N}\left(\mathrm{CH}_{3}\right)_{2}$ & $\mathrm{C}_{24} \mathrm{H}_{28} \mathrm{~N}_{4} \mathrm{O}_{2}(404.50)$ & 85 & 158 & 0.35 & 5.90 & $\begin{array}{l}71.03 \\
71.26\end{array}$ & $\begin{array}{l}7.18 \\
6.89\end{array}$ & $\begin{array}{l}13.96 \\
13.85\end{array}$ \\
\hline $2 i$ & $p-\mathrm{F}$ & $\mathrm{C}_{22} \mathrm{H}_{22} \mathrm{~F}_{2} \mathrm{~N}_{2} \mathrm{O}_{2}(384.42)$ & 85 & 158 & 0.35 & 5.86 & $\begin{array}{l}68.47 \\
68.74\end{array}$ & $\begin{array}{l}5.45 \\
5.77\end{array}$ & $\begin{array}{l}6.94 \\
7.29\end{array}$ \\
\hline $2 j$ & $o, m-\mathrm{OCH}_{2} \mathrm{O}$ & $\mathrm{C}_{26} \mathrm{H}_{24} \mathrm{~N}_{2} \mathrm{O}_{6}(436.46)$ & 85 & 158 & 0.35 & 5.50 & $\begin{array}{l}66.04 \\
66.20\end{array}$ & $\begin{array}{l}5.79 \\
5.53\end{array}$ & $\begin{array}{l}6.02 \\
6.48\end{array}$ \\
\hline
\end{tabular}

${ }^{a}$ The crystallization solvent for the solid compounds is ethanol. ${ }^{b}$ Developing solvent system is $\mathrm{CHCl}_{3} / \mathrm{CH}_{3} \mathrm{OH}(5: 3)$.

Table 3. ${ }^{1} \mathrm{H}$ NMR and mass fragmentation data of compounds (2a-j)

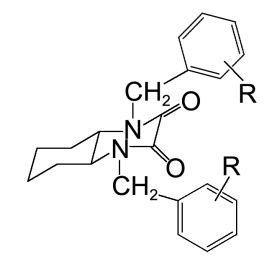

\begin{tabular}{|c|c|c|c|}
\hline $\begin{array}{c}\text { Compd. } \\
\text { No. }\end{array}$ & $\mathrm{R}$ & ${ }^{1} \mathrm{H}$ NMR & MS \\
\hline $2 a$ & $\mathrm{H}$ & $\begin{array}{l}1.00-2.90(\mathrm{~m}, 8 \mathrm{H}, c \text {-hexyl), } 3.10-3.30(\mathrm{~m}, 2 \mathrm{H}, 2 \mathrm{CH} \text { of } c \text {-hexyl), } \\
4.10-4.30 \text { and } 5.10-5.30\left(\mathrm{dd}, 4 \mathrm{H}, 2 \mathrm{CH}_{2}\right) \text {, and } 7.30(\mathrm{~s}, 10 \mathrm{H}, 2 \mathrm{Ph}) .\end{array}$ & $\mathrm{M}^{+}-1(347.47,0.3 \%)$ and $\mathrm{C}_{6} \mathrm{H}_{10}+1(85,100 \%)$ \\
\hline $2 b$ & $p-\mathrm{Br}$ & $\begin{array}{l}0.75-2.30(\mathrm{~m}, 8 \mathrm{H}, c \text {-hexyl), } 3.10-3.60(\mathrm{~m}, 2 \mathrm{H}, 2 \mathrm{CH} \text { of } c \text {-hexyl), } \\
4.00-4.50 \text { and } 4.70-5.15\left(\mathrm{dd}, 4 \mathrm{H}, 2 \mathrm{CH}_{2}\right) \text {, and } 6.85-7.50(\mathrm{~m}, 8 \mathrm{H}, 2 \\
\mathrm{Ph}) .\end{array}$ & $\begin{array}{l}\mathrm{M}^{+}(505.81,0.2 \%), \mathrm{M}^{+}+2(507.81,0.2 \%), \mathrm{M}^{+}+4 \\
(509.81,0.2 \%), \text { and } \mathrm{C}_{6} \mathrm{H}_{10}+2(86,100 \%)\end{array}$ \\
\hline $2 c$ & $p-\mathrm{Cl}$ & $\begin{array}{l}0.85-2.10(\mathrm{~m}, 8 \mathrm{H}, c \text {-hexyl), } 3.00-3.50(\mathrm{~m}, 2 \mathrm{H}, 2 \mathrm{CH} \text { of } c \text {-hexyl), } \\
4.10-4.50 \text { and } 4.70-5.20\left(\mathrm{dd}, 4 \mathrm{H}, 2 \mathrm{CH}_{2}\right) \text {, and } 6.70-7.33(\mathrm{~m}, 8 \mathrm{H}, 2 \\
\mathrm{Ph}) .\end{array}$ & $\begin{array}{l}\mathrm{M}^{+}(417.14,2.7 \%), \mathrm{M}^{+}+2(419.09,0.8 \%), \mathrm{M}^{+}-2 \mathrm{Cl} \\
(347.14,0.2 \%), \text { and } \mathrm{C}_{7} \mathrm{H}_{5} \mathrm{Cl}^{+}(124,100 \%)\end{array}$ \\
\hline 2d & $o-\mathrm{Cl}$ & $\begin{array}{l}0.90-2.10(\mathrm{~m}, 8 \mathrm{H}, c \text {-hexyl), } 3.10-3.50(\mathrm{~m}, 2 \mathrm{H}, 2 \mathrm{CH} \text { of } c \text {-hexyl), } \\
4.10-4.50 \text { and } 4.85-5.20\left(\mathrm{dd}, 4 \mathrm{H}, 2 \mathrm{CH}_{2}\right) \text {, and } 6.85-7.40(\mathrm{~m}, 8 \mathrm{H}, 2 \\
\mathrm{Ph}) \text {. }\end{array}$ & $\begin{array}{l}\mathrm{M}^{+}(417.77,4.0 \%), \mathrm{M}^{+}+2(419.10,1.4 \%), \text { and } \\
\mathrm{C}_{7} \mathrm{H}_{5} \mathrm{Cl}^{+}(124,100 \%)\end{array}$ \\
\hline $2 e$ & $p-\mathrm{CH}_{3}$ & $\begin{array}{l}0.85-2.15\left(\mathrm{~m}, 8 \mathrm{H}, c-\text { hexyl), } 2.3\left(\mathrm{~s}, 6 \mathrm{H}, 2 \mathrm{CH}_{3}\right), 3.15-3.60(\mathrm{~m}, 2 \mathrm{H} \text {, }\right. \\
2 \mathrm{CH} \text { of } c \text {-hexyl), } 4.00-4.50 \text { and } 4.90-5.15\left(\mathrm{dd}, 4 \mathrm{H}, 2 \mathrm{CH}_{2}\right) \text {, and } \\
7.15(\mathrm{~s}, 8 \mathrm{H}, 2 \mathrm{Ph}) .\end{array}$ & $\begin{array}{l}\mathrm{M}^{+}(376.35,3.8 \%), \mathrm{M}^{+}+1-2 \mathrm{CH}_{3}(347.09,16.3 \%), \\
\mathrm{M}^{+}+1-2 \mathrm{CH}_{3}(347.09,16.3 \%) .\end{array}$ \\
\hline
\end{tabular}


Table 3. Continued

\begin{tabular}{|c|c|c|c|}
\hline $\begin{array}{l}\text { Compd. } \\
\text { No. }\end{array}$ & $\mathrm{R}$ & ${ }^{1} \mathrm{H} \mathrm{NMR}$ & MS \\
\hline $2 f$ & $p-i-\mathrm{C}_{3} \mathrm{H}_{7}$ & $\begin{array}{l}0.60-2.20\left(\mathrm{~m}, 20 \mathrm{H}, c \text {-hexyl and } 4 \mathrm{CH}_{3}\right), 2.50-3.00(\mathrm{~m}, 2 \mathrm{H}, 2 \mathrm{CH}) \\
3.10-3.50(\mathrm{~m}, 2 \mathrm{H}, 2 \mathrm{CH} \text { of } c \text {-hexyl), } 4.00-4.50 \text { and } 4.80-5.20(\mathrm{dd} \text {, } \\
\left.4 \mathrm{H}, 2 \mathrm{CH}_{2}\right) \text {, and } 7.00-(\mathrm{s}, 8 \mathrm{H}, 2 \mathrm{Ph}) .\end{array}$ & $\begin{array}{l}\mathrm{M}^{+}-i-\operatorname{Pr}(376.35,3.8 \%), \mathrm{M}^{+}+1-2 \mathrm{CH}_{3}(347.09, \\
16.3 \%), \mathrm{M}^{+}+1-2 \mathrm{CH}_{3}(347.09,16.3 \%)\end{array}$ \\
\hline $2 \mathrm{~g}$ & $p-\mathrm{OCH}_{3}$ & $\begin{array}{l}0.75-2.15(\mathrm{~m}, 8 \mathrm{H}, c \text {-hexyl), } 3.00-3.20(\mathrm{~m}, 2 \mathrm{H}, 2 \mathrm{CH} \text { of } c \text {-hexyl }) \\
3.70(\mathrm{~s}, 6 \mathrm{H}, 2 \mathrm{CH} 3), 4.00-4.50 \text { and } 4.80-5.30\left(\mathrm{~m}, 4 \mathrm{H}, 2 \mathrm{CH}_{2}\right) \text {, and } \\
6.50-7.30(\mathrm{~m}, 8 \mathrm{H}, 2 \mathrm{Ph})\end{array}$ & $\begin{array}{l}\mathrm{M}^{+}+1(409.66,7.0 \%), \mathrm{M}^{+}-1(407.99,0.3 \%), p- \\
\text { pethoxybenzyl }(121.00,100 \%)\end{array}$ \\
\hline $2 \mathrm{~h}$ & $p-\mathrm{N}\left(\mathrm{CH}_{3}\right)_{2}$ & $\begin{array}{l}0.85-2.10\left(\mathrm{~m}, 8 \mathrm{H}, c \text {-hexyl), } 2.95\left(\mathrm{~s}, 12,4 \mathrm{CH}_{3}\right), 3.20-3.50(\mathrm{~m}, 2 \mathrm{H} \text {, }\right. \\
2 \mathrm{CH} \text { of } c \text {-hexyl), } 4.10-4.50 \text { and } 5.70-5.40\left(\mathrm{~m}, 4 \mathrm{H}, 2 \mathrm{CH}_{2}\right) \text {, and } \\
6.33-7.30(\mathrm{~m}, 8 \mathrm{H}, 2 \mathrm{Ph}) .\end{array}$ & $\begin{array}{l}\mathrm{M}^{+}+1-\mathrm{NMe}_{2}(391.18,1.2 \%), \mathrm{C}_{9} \mathrm{H}_{12} \mathrm{~N}^{+}(134.03 \\
100 \%)\end{array}$ \\
\hline $2 \mathbf{i}$ & $p-\mathrm{F}$ & $\begin{array}{l}0.80-2.20(\mathrm{~m}, 8 \mathrm{H}, c \text {-hexyl), } 3.10-3.60(\mathrm{~m}, 2 \mathrm{H}, 2 \mathrm{CH} \text { of } c \text {-hexyl), } \\
4.20-5.20\left(\mathrm{~m}, 4 \mathrm{H}, 2 \mathrm{CH}_{2}\right) \text {, and } 6.85-7.15(\mathrm{~m}, 8 \mathrm{H}, 2 \mathrm{Ph}-p-\mathrm{F})\end{array}$ & $\begin{array}{l}\mathrm{M}^{+}(384.05,8.0 \%), \mathrm{M}^{+}-2(382.85,45.6 \%) \\
\mathrm{C}_{7} \mathrm{H}_{4} \mathrm{~F}^{+}(105.83,100 \%)\end{array}$ \\
\hline $2 \mathbf{j}$ & $o, \mathrm{~m}-\mathrm{OCH}_{2} \mathrm{O}$ & $\begin{array}{l}0.90-2.33(\mathrm{~m}, 8 \mathrm{H}, c \text {-hexyl, }), 3.15-3.70(\mathrm{~m}, 2 \mathrm{H}, 2 \mathrm{CH} \text { of } c \text {-hexyl), } \\
4.10-4.50 \text { and } 4.85-5.30\left(\mathrm{~m}, 4 \mathrm{H}, 2 \mathrm{CH}_{2}\right), 6.00(\mathrm{~s}, 4 \mathrm{H}, 2 \mathrm{CH} 2 \text { of } \\
\text { piperonyl), and } 6.70(\mathrm{~m}, 6 \mathrm{H}, 2 \mathrm{Ph}) .\end{array}$ & $\mathrm{M}^{+}+1(437.07,1.5 \%), \mathrm{C}_{9} \mathrm{H}_{9} \mathrm{O}^{+}+1(134.96,100 \%)$ \\
\hline
\end{tabular}

borer. The test compounds (2a-j) and chloramphenicol were dissolved in dimethyl sulphoxide $(10 \mu \mathrm{mol} / \mathrm{mL})$ were loaded in the cavities. In addition, other cavities were loaded with the solvent (DMSO) and served as a negative control. The seeded plates were incubated at $28 \pm 2{ }^{\circ} \mathrm{C}$ for $48 \mathrm{~h}$. The radii of inhibition zones (in $\mathrm{mm}$ ) of triplicate sets were measured and the results are cited in Table 4.

Antifungal Activity: Compounds (2a-j) were tested for their antifungal activity in vitro, in comparison with clotrimazole as a reference drug using the standard agar cup diffusion method ${ }^{20}$ at the Assiut University Mycological Center (AUMC), Faculty of Science, Assiut University. Six pathogenic [T. rubrum (Castellani) Sabouraud AUMC 1145, and C. albicans (Robin) Berkhout AUMC 421], phytopathogenic (F. oxysporum Schlechtendal AUMC 208) and food deteriorating fungal species [A. flavus Link AUMC 3372, G. candidum Link AUMC 228, and $S$. brevicaulis (Saccardo) Bainier AUMC 363] were used in the present study. $^{23-28}$

Spore suspension in sterile distilled water was prepared from 7 days old culture of the test fungi growing on Sabouraud' dextrose broth $(30 \mathrm{~mL})$ media in $100 \mathrm{~mL}$ conical flasks. The final spore concentration was $5 \times 10^{4}$ spores $/ \mathrm{mL}$. About $15 \mathrm{~mL}$ of growth medium was introduced on sterilized Petri dishes of $10 \mathrm{~cm}$ diameter and inoculated with $1 \mathrm{~mL}$ of spore suspension. Plates were shaken gently to homogenize the inocula. Antifungal activity of the test compounds (2a-j) was performed by the standard agar cup diffusion method as follow:

After solidification of the media, $5 \mathrm{~mm}$ cavities were cut in the solidified agar (4 cavities/plate) using sterile cork borer and was filled with the solutions of the test compounds and cotrimazole $(10 \mu \mathrm{mol} / \mathrm{mL}$ in DMSO). In addition, other cavities were impregnated with the solvent (DMSO) and served as a negative control. The seeded plates were incubated at $28 \pm 2{ }^{\circ} \mathrm{C}$ for 7 days. The radii of inhibition zones (in $\mathrm{mm}$ ) of triplicate sets were measured at successive intervals during the incubation period and the results are cited in Table 5 .
The Minimum Inhibitory Concentrations (MICs). The test compounds giving positive results were diluted with DMSO to prepare a series of descending concentration down to $0.15 \mu \mathrm{mol} / \mathrm{mL}$. Diluted solutions were similarly assayed as mentioned before and the least concentration (below which no activity) was recorded. The squares of inhibition zone diameters were plotted against log concentrations of the tested compounds, extrapolation of the resulting straight line to intersect with $\log$ concentration scale in the curve corresponded to $\log$ MIC, and MIC was obtained as antilog, and the results are cited in Tables 6 and 7.

\section{Results and Discussion}

Chemistry. Compounds (la-j) were prepared according to reported procedures. ${ }^{16}$ Target compounds, 1,4-disubstituted octahydroquinoxaline-2,3-dione derivatives (2a-j) were prepared by fussing compounds (1a-j) and freshly distilled diethyl oxalate. Structures of compounds, $(\mathbf{2} \mathbf{a}-\mathbf{j})$ were confirmed by IR, ${ }^{1} \mathrm{H}-\mathrm{NMR}$, and MS, in addition to elemental method of analyses. IR spectra of compounds (2a-j) were characterized by lack of the characteristic bands due to $\mathrm{NH}$ functions of the intermediates (1a-j) and exhibited bands attributed to $\mathrm{C}=\mathrm{O}$ stretching function at $1676-1620 \mathrm{~cm}^{-1}$. ${ }^{1} \mathrm{H}-\mathrm{NMR}$ spectra of compounds (1a-j), Table 1 exhibited a general pattern for the cyclohexyl moiety and $2 \mathrm{NH}$ (exchangeable with $\mathrm{D}_{2} \mathrm{O}$ ) groups which is characterized as a multiplet signal between 0.70 and $2.40 \mathrm{ppm}$. The benzylic $\mathrm{CH}_{2}$ protons appeared as a doublet of doublet or multiplet around 3.20 and 4.00 ppm. In addition, the specific function groups appeared in positions that are in accordance with the structures. Compound 1e showed a singlet signal equivalent to 6 protons at $2.33 \mathrm{ppm}$ corresponding to two $p-\mathrm{CH}_{3}$ groups. Moreover, the aromatic protons showed patterns at positions which are in accordance to the structures of the target compounds (1a-j). ${ }^{1} \mathrm{H}-\mathrm{NMR}$ spectra of compounds $(\mathbf{2} \mathbf{a}-\mathbf{j})$, Table 3 revealed absence of signals corresponding to the exchangeable protons of the $2 \mathrm{NH}$ groups proved the process of cyclization. In addition, the spectra showed a general pattern for the cyclohexyl 
Table 4. Antibacterial activity of compounds $\mathbf{2 a - j}$ and chloramphenicol (inhibition zone in $\mathrm{mm}$ )

\begin{tabular}{|c|c|c|c|c|c|c|c|c|c|c|c|c|c|c|c|c|c|c|c|c|c|c|c|c|}
\hline \multirow[b]{2}{*}{ Organisms } & \multicolumn{5}{|c|}{$2 a$} & \multicolumn{3}{|c|}{$2 b$} & \multicolumn{5}{|c|}{$2 \mathrm{c}$} & \multicolumn{6}{|c|}{$2 d$} & \multicolumn{5}{|c|}{$2 e$} \\
\hline & 10 & 5 & 2.5 & 1.25 & 0.6 & 10 & 5 & 2.5 & 10 & 52 & 2.51 & 1.250 & 0.6 & 10 & 52 & 2.51. & 1.250 & 0.60 & 0.3 & 10 & 5 & 2.5 & 1.25 & 0.6 \\
\hline $\begin{array}{l}\text { Serratia marcescens (-ve) } \\
\text { AUMC No. B-55 }\end{array}$ & 11 & 0 & - & - & - & 10 & 8 & 0 & 8 & 8 & 0 & - & - & 101 & 10 & 8 & 0 & - & - & 12 & 8 & 0 & - & - \\
\hline $\begin{array}{l}\text { Pseudomonas aeruginosa (-ve) } \\
\text { AUMC No. B-73 }\end{array}$ & 8 & 8 & 0 & - & - & 0 & - & - & 0 & - & - & - & - & 0 & - & - & - & - & - & 13 & 10 & 0 & - & - \\
\hline $\begin{array}{l}\text { Escherichia coli (-ve) } \\
\text { AUMC No. B-53 }\end{array}$ & 17 & 0 & - & - & - & 14 & 0 & - & 211 & 15 & 0 & - & & 121 & 12 & 10 & 0 & - & - & 19 & 12 & 10 & 0 & - \\
\hline $\begin{array}{l}\text { Staphylococcus aureus }(+\mathrm{ve}) \\
\text { AUMC No. B-54 }\end{array}$ & 17 & 13 & 10 & 8 & 0 & 13 & 0 & - & 191 & 17 & 13 & 8 & 0 & 151 & 15 & 121 & 10 & 0 & -1 & 18 & 14 & 10 & 8 & 0 \\
\hline $\begin{array}{l}\text { Bacillus cereus }(+\mathrm{ve}) \\
\text { AUMC No B-52 }\end{array}$ & 18 & 14 & 10 & 8 & 0 & 23 & 14 & 0 & 251 & 161 & 10 & 0 & - & 151 & 15 & 141 & 121 & 10 & 02 & 24 & 14 & 10 & 0 & - \\
\hline $\begin{array}{l}\text { Micrococcus luteus } \\
\text { AUMC No B-112 }\end{array}$ & 23 & 17 & 11 & 0 & - & 15 & 8 & 0 & 221 & 181 & 13 & 0 & - & 181 & 18 & 171 & 13 & 0 & - & 21 & 18 & 13 & 0 & - \\
\hline
\end{tabular}

\begin{tabular}{|c|c|c|c|c|c|c|c|c|c|c|c|c|c|c|c|c|c|c|c|c|c|c|c|}
\hline \multirow{2}{*}{ 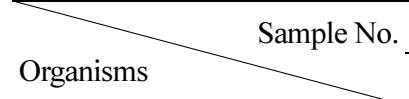 } & \multicolumn{6}{|c|}{$2 f$} & \multicolumn{6}{|c|}{$2 \mathrm{~g}$} & \multicolumn{6}{|c|}{$2 \mathrm{~h}$} & \multicolumn{5}{|c|}{$2 i$} \\
\hline & 10 & 5 & 2.5 & 1.25 & 0.6 & 0.3 & 10 & 5 & 2.5 & 1.25 & 0.6 & 0.3 & 10 & 5 & 2.51 & 1.250 & 0.6 & 0.3 & 10 & 5 & 2.5 & 1.25 & 06 \\
\hline $\begin{array}{l}\text { Serratia marcescens (-ve) } \\
\text { AUMC No. B-55 }\end{array}$ & 0 & - & - & - & - & - & 0 & - & - & - & - & - & 0 & - & - & - & - & - & 11 & 8 & 0 & - & - \\
\hline $\begin{array}{l}\text { Pseudomonas aeruginosa (-ve) } \\
\text { AUMC No. B-73 }\end{array}$ & 0 & - & - & - & - & - & 0 & - & - & - & - & - & 0 & - & - & - & - & - & 8 & 8 & 0 & - & - \\
\hline $\begin{array}{l}\text { Escherichia coli (-ve) } \\
\text { AUMC No. B-53 }\end{array}$ & 14 & 14 & 12 & 11 & 0 & - & 8 & 0 & - & - & - & - & 16 & 12 & 10 & 0 & - & - & 15 & 12 & 10 & 8 & 0 \\
\hline $\begin{array}{l}\text { Staphylococcus aureus (+ve) } \\
\text { AUMC No. B-54 }\end{array}$ & 15 & 15 & 12 & 10 & 0 & - & 8 & 8 & 0 & - & - & - & 16 & 15 & 13 & 11 & 8 & 0 & 16 & 14 & 10 & 8 & 0 \\
\hline $\begin{array}{l}\text { Bacillus cereus }(+\mathrm{ve}) \\
\text { AUMC NoB-52 }\end{array}$ & 22 & 22 & 20 & 16 & 12 & 0 & 14 & 14 & 14 & 12 & 12 & 0 & 21 & 18 & 14 & 13 & 8 & 0 & 16 & 12 & 8 & 0 & - \\
\hline $\begin{array}{l}\text { Micrococcus luteus } \\
\text { AUMC No B-112 }\end{array}$ & 18 & 18 & 15 & 14 & 0 & - & 8 & 0 & - & - & - & - & 18 & 18 & 17 & 13 & 0 & - & 18 & 17 & 8 & 0 & - \\
\hline
\end{tabular}

AUMC No B-112

\begin{tabular}{|c|c|c|c|c|c|c|c|c|c|c|c|}
\hline \multirow[b]{2}{*}{ Organisms } & \multicolumn{5}{|c|}{$2 \mathrm{j}$} & \multicolumn{6}{|c|}{ Refer. } \\
\hline & 10 & 5 & 2.5 & 1.25 & 0.6 & 10 & 5 & 2.5 & 1.25 & 0.6 & 0.3 \\
\hline $\begin{array}{l}\text { Serratia marcescens (-ve) } \\
\text { AUMC No. B-55 }\end{array}$ & 8 & 0 & - & - & - & 41 & 40 & 38 & 34 & 28 & 26 \\
\hline $\begin{array}{l}\text { Pseudomonas aeruginosa (-ve) } \\
\text { AUMC No. B-73 }\end{array}$ & 13 & 11 & 0 & - & - & 16 & 14 & 12 & 12 & 10 & 10 \\
\hline $\begin{array}{l}\text { Escherichia coli (-ve) } \\
\text { AUMC No. B-53 }\end{array}$ & 14 & 8 & 0 & - & - & 30 & 26 & 26 & 20 & 16 & 14 \\
\hline $\begin{array}{l}\text { Staphylococcus aureus (+ve) } \\
\text { AUMC No. B-54 }\end{array}$ & 18 & 11 & 0 & - & - & 17 & 17 & 15 & 13 & 12 & 12 \\
\hline $\begin{array}{l}\text { Bacillus cereus }(+\mathrm{ve}) \\
\text { AUMC NoB-52 }\end{array}$ & 18 & 14 & 12 & 8 & 0 & 34 & 34 & 32 & 30 & 28 & 25 \\
\hline $\begin{array}{l}\text { Micrococcus luteus } \\
\text { AUMC No B-112 }\end{array}$ & 22 & 0 & - & - & - & 20 & 14 & 12 & 0 & - & - \\
\hline
\end{tabular}

Refer. $=$ Chloramphenicol as antibacterial standard. AUMC $=$ Assiut University Mycological Center

moiety as a broad multiplet signal at 0.60 and $2.90 \mathrm{ppm}$. The spectra of the target compounds (2a-j) showed a characteristic multiplet equivalent to 2 protons of the methine protons of the cyclohexyl moiety at 3.00 and $3.60 \mathrm{ppm}$. Also, they showed doublet of doublet at 4.25 and $5.12 \mathrm{ppm}$ corresponding to the benzylic $2 \mathrm{CH}_{2}$ groups. MS of compound (2a-j) revealed the molecular ion peaks $\mathrm{M}^{+}$corresponding to the molecular weight for compounds $\mathbf{2 b}, \mathbf{2 c}, \mathbf{2 d}, \mathbf{2 e}$, and $\mathbf{2 i}$ and $\mathrm{M}^{+}+1$ for compounds $2 \mathbf{g}$ and $\mathbf{2} \mathbf{j}, \mathbf{M}^{+}-1$ for compound $2 \mathbf{a}, \mathbf{M}^{+}-i$-Pr for compound $\mathbf{2 f}$ and $\mathrm{M}^{+}+1-\mathrm{NMe}_{2}$ for compound $\mathbf{2 h}$.

Lipophilicity. The lipophilicity of the target compounds $\mathbf{2 a - j}$ is expressed in the term of Clog P values (Table 2). The values were computed with a routine method called calculated $\log \mathrm{P}(\mathrm{Clog} \mathrm{P})$ contained in a PC-software package as mentioned under the experimental section $^{18}$ The target compounds exhibited high values for Clog P, Table 2. This work devoted to study the effect of the lipophilicity on the antimicrobial activity.

X-ray Diffraction (XRD). The X-ray diffraction (XRD) is based on observing the scattered intensity of an X-ray beam hitting a sample as a function of incident and scattered angle, polarization and wave length or energy. On the other 
Table 5. Antifungal activity of compounds $\mathbf{2 a - j}$ and clotrizmazole (inhibition zone in $\mathrm{mm}$ )

\begin{tabular}{|c|c|c|c|c|c|c|c|c|c|c|c|c|c|c|c|c|c|c|c|c|c|c|c|c|c|}
\hline \multirow[b]{2}{*}{ Organisms } & \multicolumn{5}{|c|}{$2 a$} & \multicolumn{4}{|c|}{$2 b$} & \multicolumn{4}{|c|}{$2 \mathrm{c}$} & \multicolumn{5}{|c|}{ 2d } & \multicolumn{7}{|c|}{$2 \mathrm{e}$} \\
\hline & 10 & 5 & 2.5 & 1.25 & 0.6 & 10 & 5 & 2.5 & 1.25 & 10 & 5 & 2.5 & 1.25 & 10 & 5 & 2.51 .25 & 50.6 & 0.3 & 10 & 5 & 2.5 & 1.25 & 0.6 & 0.3 & 0.15 \\
\hline $\begin{array}{l}\text { Candida albicans } \\
\text { AUMC No. } 418\end{array}$ & 12 & 8 & 8 & 0 & - & 8 & 0 & - & - & 14 & 10 & 0 & - & 14 & 14 & 120 & - & - & 12 & 12 & 0 & - & - & - & - \\
\hline Geotrichum candidum & 14 & 13 & 12 & 10 & 0 & 18 & 12 & 8 & 0 & 19 & 13 & 12 & 0 & 20 & 20 & 1614 & 12 & 0 & 16 & 15 & 14 & 13 & 10 & 8 & 0 \\
\hline
\end{tabular}

AUMC No. 226

Fusarium oxysporum

AUMC No. 5119

Aspergillus flavus

AUMC No. 1276

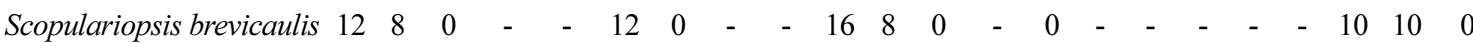

AUMC No. 729

Trichophyton rubrum

$13 \quad 12 \quad 10 \quad 0$

0

AUMC No. 1804

\begin{tabular}{|c|c|c|c|c|c|c|c|c|c|c|c|c|c|c|c|c|c|c|}
\hline \multirow[b]{2}{*}{ Organisms } & \multicolumn{5}{|c|}{$2 f$} & \multicolumn{4}{|c|}{$2 \mathrm{~g}$} & \multicolumn{4}{|c|}{$2 h$} & \multicolumn{5}{|c|}{$2 \mathrm{i}$} \\
\hline & 10 & 5 & 2.5 & 1.25 & 0.6 & 0.3 & 10 & 5 & 10 & 5 & 2.5 & 1.25 & 0.6 & 0.3 & 10 & 5 & 2.5 & 0.125 \\
\hline $\begin{array}{l}\text { Candida albicans } \\
\text { AUMC No. } 418\end{array}$ & 23 & 14 & 13 & 10 & 0 & - & 0 & - & 18 & 13 & 12 & 0 & - & - & 13 & 8 & 0 & - \\
\hline $\begin{array}{l}\text { Geotrichum candidum } \\
\text { AUMC No. } 226\end{array}$ & 24 & 20 & 16 & 14 & 12 & 0 & 12 & 0 & 22 & 20 & 16 & 14 & 10 & 0 & 14 & 0 & - & - \\
\hline $\begin{array}{l}\text { Fusarium oxysporum } \\
\text { AUMC No. } 5119\end{array}$ & 18 & 18 & 14 & 12 & 10 & 0 & 0 & - & 15 & 15 & 13 & 12 & 10 & 0 & 12 & 10 & 8 & 0 \\
\hline $\begin{array}{l}\text { Aspergillus flavus } \\
\text { AUMC No. } 1276\end{array}$ & 14 & 11 & 0 & - & - & - & 0 & - & 13 & 10 & 0 & - & - & - & 0 & - & - & - \\
\hline $\begin{array}{l}\text { Scopulariopsis brevicaulis } \\
\text { AUMC No. } 729\end{array}$ & 20 & 14 & 10 & 0 & - & - & 0 & - & 20 & 17 & 13 & 12 & 0 & - & 10 & 10 & 0 & - \\
\hline $\begin{array}{l}\text { Trichophyton rubrum } \\
\text { AUMC No. } 1804\end{array}$ & 28 & 22 & 18 & 8 & 0 & - & 0 & - & 28 & 22 & 18 & 10 & 0 & - & 13 & 0 & - & - \\
\hline
\end{tabular}

AUMC No. 1804

\begin{tabular}{|c|c|c|c|c|c|c|c|c|c|c|c|}
\hline \multirow{2}{*}{ 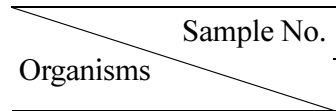 } & \multicolumn{4}{|c|}{$2 j$} & \multicolumn{7}{|c|}{ Refer. } \\
\hline & 10 & 5 & 2.5 & 1.25 & 10 & 5 & 2.5 & 1.25 & 0.6 & 0.3 & 0.15 \\
\hline $\begin{array}{l}\text { Candida albicans } \\
\text { AUMC No. } 418\end{array}$ & 12 & 0 & - & - & 30 & 30 & 30 & 26 & 26 & 26 & 20 \\
\hline $\begin{array}{l}\text { Geotrichum candidum } \\
\text { AUMC No. } 226\end{array}$ & 14 & 12 & 12 & 0 & 24 & 22 & 22 & 22 & 22 & 22 & 20 \\
\hline $\begin{array}{l}\text { Fusarium oxysporum } \\
\text { AUMC No. } 5119\end{array}$ & 0 & - & - & - & 22 & 22 & 22 & 22 & 18 & 18 & 16 \\
\hline $\begin{array}{l}\text { Aspergillus flavus } \\
\text { AUMC No. } 1276\end{array}$ & 0 & - & - & - & 27 & 27 & 27 & 25 & 25 & 25 & 25 \\
\hline $\begin{array}{l}\text { Scopulariopsis brevicaulis } \\
\text { AUMC No. } 729\end{array}$ & 0 & - & - & - & 26 & 24 & 23 & 23 & 20 & 20 & 17 \\
\hline $\begin{array}{l}\text { Trichophyton rubrum } \\
\text { AUMC No. } 1804\end{array}$ & 14 & 0 & - & - & 35 & 34 & 34 & 34 & 34 & 34 & 34 \\
\hline
\end{tabular}

Refer. $=$ clotrizmazole as antifungal standard. AUMC $=$ Assiut University Mycological Center

hand, to identify unknown substance by comparing diffraction data against a data base maintained by the international centre for diffraction data. ${ }^{19}$ The latter contains no XRD data about the new compounds (2a-j). The X-ray diffraction spectra for compounds $\mathbf{2 b}$ and $\mathbf{2 e}$ are rich in bands corresponding to $\mathrm{C}, \mathrm{H}, \mathrm{Br}$, and $\mathrm{N}$.

Antimicrobial Activity.

Antibacterial Activity: The test compounds (2a-j) were assayed using the standard agar cup diffusion method ${ }^{20}$ at a concentration of $10 \mu \mathrm{mol} / \mathrm{mL}$ and those giving positive results were diluted with DMSO to prepare a series of descending concentrations down to $0.15 \mu \mathrm{mol} / \mathrm{mL}$ and were similarly assayed and the least concentration (below which no activity) was recorded as the MIC.

Results of the antibacterial activity, Table 4, indicated that at a concentration of $10 \mu \mathrm{mol} / \mathrm{mL}$ most of the test compounds were active against most of the used bacterial strains. Compounds $\mathbf{2 b}, \mathbf{2 c}$, and $\mathbf{2 d}$ were inactive against $P$. aeruginosa and compounds $\mathbf{2 f}, \mathbf{2 g}$, and $\mathbf{2 h}$ were inactive against $S$. Marcescens and P. aeruginosa. In addition the test compounds 
Table 6. Antibacterial activity (inhibition zone in $\mathrm{mm}$ and MICs given in brackets at $10 \mu \mathrm{mol} / \mathrm{mL}$ ) of compounds (2a-j) and chloramphenicol

\begin{tabular}{ccccccc}
\hline Sample No. & Serratia marcescens & $\begin{array}{c}\text { Pseudomonas } \\
\text { aeruginosa }\end{array}$ & Escherichia coli & $\begin{array}{c}\text { Staphylococcus } \\
\text { aureus }\end{array}$ & Bacillus cereus & Micrococcus luteus \\
\hline $\mathbf{2 a}$ & $11(5)$ & $8(2.5)$ & $17(5.0)$ & $17(0.9)$ & $18(0.98)$ & $23(1.75)$ \\
$\mathbf{2 b}$ & $10(2.5)$ & - & $14(5.0)$ & $13(5.0)$ & $23(3.32)$ & $15(3.8)$ \\
$\mathbf{2}$ & $8(2.5)$ & - & $21(2.5)$ & $19(0.8)$ & $25(2.2)$ & $22(1.25)$ \\
$\mathbf{2 d}$ & $10(1.25)$ & - & $12(1.25)$ & $15(0.6)$ & $15(0.3)$ & $18(0.6)$ \\
$\mathbf{2}$ & $12(2.87)$ & $13(2.5)$ & $19(1.96)$ & $18(0.98)$ & $24(2.3)$ & $21(1.25)$ \\
$\mathbf{2}$ & - & - & $14(0.6)$ & $15(0.6)$ & $22(0.3)$ & $18(0.6)$ \\
$\mathbf{2}$ & - & - & $8(5.0)$ & $8(2.5)$ & $14(0.3)$ & $8(2.5)$ \\
$\mathbf{2}$ & - & - & $16(1.25)$ & $8(1.25)$ & $21(0.42)$ & $18(2.5)$ \\
$\mathbf{2}$ & $11(2.5)$ & $8(2.5)$ & $15(0.65)$ & $16(0.3)$ & $18(1.65)$ & $18(0.5)$ \\
$\mathbf{2}$ & $8(5.0)$ & $13(2.5)$ & $14(3.57)$ & $18(3.3)$ & $18(0.81)$ & $22(5.0)$ \\
Refer. & $41(0.04)$ & $16(0.07)$ & $30(0.16)$ & $17(0.04)$ & $34(0.006)$ & $20(1.51)$ \\
\hline
\end{tabular}

Table 7. Antifungal activity (inhibition zone in $\mathrm{mm}$ and MICs given in brackets at $10 \mu \mathrm{mol} / \mathrm{mL}$ ) of test compounds (2a-j) and clotrizmazole

\begin{tabular}{ccccccc}
\hline Sample No. & Candida albicans & $\begin{array}{c}\text { Geotrichum } \\
\text { candidum }\end{array}$ & $\begin{array}{c}\text { Fusarium } \\
\text { oxysporum }\end{array}$ & Aspergillus flavus & $\begin{array}{c}\text { Scopulariopsis } \\
\text { brevicaulis }\end{array}$ & $\begin{array}{c}\text { Trichophyton } \\
\text { rubrum }\end{array}$ \\
\hline $\mathbf{2 a}$ & $12(1.54)$ & $14(0.6)$ & $13(1.25)$ & $8(2.5)$ & $12(2.87)$ & $12(1.25)$ \\
$\mathbf{2 b}$ & $8(5.0)$ & $18(2.0)$ & - & - & $12(5.0)$ & $18(4.21)$ \\
$\mathbf{2}$ & $14(2.5)$ & $19(1.5)$ & $17(1.52)$ & $14(3.57)$ & $16(3.96)$ & $25(3.86)$ \\
$\mathbf{2 d}$ & $14(1.25)$ & $20(0.3)$ & - & $12(1.25)$ & - & $20(0.64)$ \\
$\mathbf{2} \mathbf{2}$ & $12(2.5)$ & $16(0.15)$ & - & $8(5.0)$ & $10(2.5)$ & $20(3.96)$ \\
$\mathbf{2}$ & $23(1.0)$ & $24(0.36)$ & $18(0.3)$ & $14(2.5)$ & $20(1.79)$ & $28(0.90)$ \\
$\mathbf{2} \mathbf{g}$ & - & $12(5.0)$ & - & - & - & - \\
$\mathbf{2}$ & $18(1.25)$ & $22(0.32)$ & $15(0.3)$ & $13(2.5)$ & $20(0.6)$ & $28(0.96)$ \\
$\mathbf{2}$ & $13(3.28)$ & $14(5.0)$ & $12(1.25)$ & - & $10(2.5)$ & $13(5.0)$ \\
$\mathbf{2} \mathbf{j}$ & $12(5.0)$ & $14(1.25)$ & - & - & - & $14(5.0)$ \\
Refer. & $30(0.005)$ & $24(0.012)$ & $22(0.005)$ & $27(0.17)$ & $26(0.005)$ & $35(0.003)$ \\
\hline
\end{tabular}

(2a-j) showed 19.5-29.5\% of the antibacterial activity of chloramphenicol against $S$. Marcescens, 50.0-81.5\% against S. Marcescens, 40.0-63.5\% against E. coli, 47.1-106.0\% against $S$. aureus, $41.5-70.5 \%$ against B. Cereus, and $40.0-$ $115.0 \%$ against $M$. Luteus. Moreover the variation of the antibacterial activity with concentrations was indicated in Table 4. It was noted that the most sensitive organisms to the test compounds were $S$. aureus, B. cereus, E. coli and $M$. Luteus. It is noteworthy to mention that, compounds $\mathbf{2 a}, \mathbf{2 c}$, 2e, $2 \mathbf{i}$, and $2 \mathbf{j}$ bearing $\mathrm{H}, p-\mathrm{Cl}, p-\mathrm{CH}_{3}, p-\mathrm{F}$, and piperonyl moieties respectively gave antibacterial activities up to concentrations of $1.25 \mu \mathrm{mol} / \mathrm{mL}$. The most active derivatives of the target compounds are $\mathbf{2 d}, \mathbf{2} \mathbf{f}, \mathbf{2} \mathbf{g}$, and $\mathbf{2 h}$ bearing in their structures $o-\mathrm{Cl}, p-i \mathrm{Pr}, p-\mathrm{OCH}_{3}$, and $p-\mathrm{NMe}_{2}$ moieties giving antibacterial activities at concentrations of $0.6 \mu \mathrm{mol} / \mathrm{mL}$.

Antifungal Activity: Results of the antifungal activity, Table 5 revealed that the test compounds showed variable activities against the used fungal strains in comparison to clotrimazole as a reference drug. On the other hand, compound 2b was inactive against $F$. oxysporum, and A. flavous, compound 2d was inactive against $F$. oxysporum, and $S$. brevicaulius, compound $\mathbf{2 e}$ was inactive against $F$. oxysporum, and compound $\mathbf{2} \mathbf{i}$ was inactive against $A$. flavous. Moreover, compound $\mathbf{2 g}$ was nearly completely inactive against the used fungal strains.

In addition the test compounds (2a-j) showed 60.0$100.0 \%$ of the antifungal activity of clotrimazole against $C$. albicans, 58.5-100.0\% against $G$. Candidum, 59.0-82.5\% against $F$. oxysporum, 30.0-52.0\% against A. flavus, 38.5$91.0 \%$ against $S$. bervicularis, and $34.4-80.0 \%$ against $T$. rubrum. Moreover the variation of the antifungal activity with concentrations was indicated in Table 6. It was noted that, most of the tested compounds showed effective antifungal activity against the used fungi. Also some test compounds were more active than clotrimazole against $F$. oxysporum. Again, It is noteworthy to mention that, the least active antifungal compound was $2 \mathrm{~g}(\mathrm{R}=\mathrm{OMe})$ giving activity at $10.0 \mu \mathrm{mol} / \mathrm{mL}$, while compounds $\mathbf{2 b}, \mathbf{2 c}, \mathbf{2} \mathbf{i}$, and $\mathbf{2} \mathbf{j}$ bearing $p$ - $\mathrm{Br}, p-\mathrm{Cl}, p-\mathrm{F}$, and piperonyl moieties respectively gave antifungal activity at $2.5 \mu \mathrm{mol} / \mathrm{mL}$. Compound $2 \mathrm{a}(\mathrm{R}=\mathrm{H})$ showed antifungal activity at $1.25 \mu \mathrm{mol} / \mathrm{mL}$. On the other hand, compounds $\mathbf{2 d}, \mathbf{2 f}$, and $\mathbf{2 h}$ bearing in their structures $o$ $\mathrm{Cl}, p$-iPr, and $p-\mathrm{NMe}_{2}$ moieties respectively gave antifungal activities at concentrations of $0.3 \mu \mathrm{mol} / \mathrm{mL}$.

\section{Conclusion}

A number of 1,4-disubstituted octahydroquinoxaline-2,3- 
dione derivatives were prepared through two steps reaction. This protocol involves the formation of $N, N$-disubstituted cyclohexane-1,2-diamine derivatives (1a-j) followed by cyclization with diethyl oxalate. The structures of the target compounds, 1,4-disubstituted octahydroquinoxaline-2,3-dione derivatives (2a-j) were elucidated depending upon different spectral data as well as the elemental methods of analyses. In addition, mass fragmentation and X-ray diffraction analyses were carried out. Moreover, the lipophilicity of the target compounds as expressed from the Clog $\mathrm{P}$ and the measured $\mathrm{R}_{\mathrm{f}}$ values were cited. The antimicrobial and MIC activities of compounds (2a-j) were investigated. Most of the test compounds showed weak to moderate antibacterial activity in comparison to chloramphenicol as a reference drug. The most active compounds were $\mathbf{2 d}, \mathbf{2 f}, \mathbf{2 g}$, and $\mathbf{2 h}$ with $o-\mathrm{Cl}, p$ $i \mathrm{Pr}, p-\mathrm{OCH}_{3}$, and $p-\mathrm{NMe}_{2}$ moieties giving activities at concentrations $0.6 \mu \mathrm{mol} / \mathrm{mL}$. Also some test compounds were more active than clotrimazole with regards to their antifugal activities. Compounds $\mathbf{2 d}, \mathbf{2 f}$, and $\mathbf{2 h}$ with $o-\mathrm{Cl}, p-i \mathrm{Pr}$, and $p$ $\mathrm{NMe}_{2}$ moieties respectively gave antifungal activities at concentrations $0.3 \mu \mathrm{mol} / \mathrm{mL}$.

\section{References}

1. Sensi, P.; Gialdroni, C. Burger's Medicinal Chemistry and Drug Discovery; $6^{\text {th }}$ ed.; John Wiley and Sons: New York, 2003; Vol. 5, p 807.

2. Lipsitch, M. Trends in Microbiology "The Rise and Full of Antimicrobial Resistance", 2001; p 438.

3. Donald, P. R.; Schaaf, H. S. Pediatric Resp. Rev. 2007, 8, 124.

4. Ramalingam, P.; Ganapaty, S.; Rao, Ch. B. Bioorg. Med. Chem. Lett. 2010, 20, 406

5. Diel, A.; William, D. H.; Morris, H. R.; Smith, G. A.; Freeney, J.; Roberts, G. C. K. J. Am. Chem. Soc. 1975, 97, 2497.

6. Batlly, C.; Echepare, S.; Gago, F.; Wanng, M. J. Anticancer Drug Res. Chem. 1999, 15, 270.
7. Davey, P. G. "Antimicrobial Chemotherapy" in "Concise Textbook of Medicine", Oxford University press: 2000; p 1475.

8. Khosarkar, A. S.; Shinde, D. B. Bioorg. Med. Chem. Lett. 2006, 16,6181 .

9. Ali, A. Y.; EzzEl-Din, M. S.; Hasananen, J. A.; Abdel-Fatah, M. E. Ind. J. Chem. 2003, 42B, 2835.

10. Renult, J.; Baron, M.; Mailiet, P. Eur. J. Med. Chem. 1981, 16(6), 545.

11. Sarges, R.; Howard, H. R.; Browne, R. G.; Label, L. A.; Seymour, P. A.; Koe, B. K. J. Med. Chem. 2007, 33(8), 2240.

12. Majid, M.; Bakhtiari, K.; Tehrani, M. H.; Javadi, N. M.; Oskooie, H. A. Arkivoc 2006, xvi, 16.

13. Sheardown, M. J.; Nielsen, E. O.; Hansen, A. J.; Jacobsen, P.; Honore, J. Science 1990, 247(4942), 541.

14. El-Wahy, H. M. A. Tetrahedron 2000, 56, 897.

15. Abuel-Magd, A. A.; El-Shorbagi, A. A.; Hussein, M. A.; Hamdy, M. M.; Abdel-Alim, A. M. Bulletin of Pharmaceutical Sciences 2004, 27(2), 193.

16. Hussein, M. A.; Diab, A. K. Bulletin of Pharmaceutical Sciences 2005, 28(1), 37.

17. Hussein, M. A.; Hashem, M. Arch. Pharm. Pharm. Med. Chem. 2008, $341(6), 370$.

18. Leo, A. J. Chem. Rev. 2001, 93, 1281.

19. Batterman, B. W.; Cole, H. Reviews of Moderm Physics 1964, $36(3), 681$.

20. William, H. Microbiological Assay, An Introduction to Quantitative Principles and Evaluation; Academic press: New York, 1977.

21. Jain, S. R.; Kar, A. Planta Med. 1971, 20, 118.

22. Out, S. A.; Sherif, S. M. Folia Microbiol. 1993, 38(3), 181.

23. Moubasher, A. A. Soil Fungi in Qatar and Other Arab Countries; Scientific and Applied Research Center: University of Qatar, Qatar, 1993.

24. West, T. P. F. Microbios 1993, 56, 226, 27.

25. Kwion-Chung, K. J.; Bennett, J. E. Medical Mycology; Leo \& Febiger: Philadelphia, 1992; p 866.

26. Moubasher, A. A.; Mazen, M. B.; Moharram, A. M.; EI-Shanawany, A. Proc. $10^{\text {th }}$ Ann. Sci. Coni. April, 1992.

27. Mazen, M. B.; Abdel-Malek, A. Y.; Allam, A. D.; Hashem, M. Bul. Fac. Sci. Asiut Univ. 1997, 25(I-D), 67.

28. Hashem, M. Czech Mycology 2002, 56(3-4), 223. 